\title{
The Design of Muzzle Velocity Measuring System for Rapid Rate Firing Gun
}

\author{
Yuchen Yue, Fuquan Zhao, Jinzhong Zhang and Xiaoping Han \\ Academy of Armored Force Engineering, Department of Arms Engineering, Beijing 100072, China
}

\begin{abstract}
The paper focus on the problem that a certain type of rapid rate firing gun, which the army equipped, it's muzzle velocity is difficult to measure. On the basis of summing up the advantages and disadvantages of all kinds of muzzle velocity measurement method, the paper put forward the phase measurement method of muzzle velocity, and thus design a muzzle velocity measuring system. First, the paper elaborated the basic principle of phase measurement and comprehensive use of integrated metal close detector CS209A and analog PLL NE564 to build up the speed measurement system hardware. When the projectile pass through the coil that fixed at the muzzle, which has the fixed distance, the two coil which connected with the two CS209A will respectively produce different phase of two signals input NE564. Then the NE564 outputs the phase difference signal to the upper computer software which written by the LabVIEW and calculation of projectile muzzle velocity. The results show that the speed measurement system is accurate and reliable, and it is suitable for the popularization of the troops.
\end{abstract}

KEYWORD: phase difference method; muzzle initial velocity; dynamic measure

\section{INTRODUCTION}

Muzzle velocity is a key parameter of the artillery $\mathrm{R}$ $\& \mathrm{D}$, firing table preparation, researching on interior, exterior and terminal ballistic performance, testing and evaluating of gun system overall performance and lethality (LI Xiao-yin). Based on the real-time and accurate measure the muzzle velocity can realize for exterior ballistic pre-calculation, improved estimation precision of solving hit problem, so as to improve maximize hit rate, and it's also the key of projectile intelligent control and achieve precision strike in the future (CHU Ling-yue, 2010). Nowadays, muzzle velocity measurement methods have currently used as sky screen method, coil target method and light screen method (Wang Ke, 2015). Those methods through practical application for a long time has been ultimate mature, and have high precision with oneshot gun muzzle velocity measurement. However, there are still the following questions (HUANG Xuegong, 2006): Firstly, the hardware of those equipment all have large volume and inconvenient to operate; Secondly, due to the rapid rate of firing artillery exist the problems of high projectile RF and muzzle continuous flame that lead to the testing result's accuracy is unsatisfactory; Thirdly, those methods mentioned above are external measurement methods so that are not really obtain projectile velocity at the muzzle.
This article in order to solving the above three problems designed a new muzzle velocity measurement system which applicate to rapid rate firing gun which using the phase measurement method. Comprehensive use the metal close detector CS209A and highspeed PLL NE564 to detect real-time projectile phase. Collect the projectile's phase difference signal pass through fixed distance, and then processing the data to get the muzzle velocity. The new system has the advantages of small volume and can be easily installed at the muzzle position. The continuous firing flame and the influence of environment are small.

\section{THE BASIC PRINCIPLE}

The muzzle velocity measurement system using the area cutting theory, the structure principle diagram is shown in figure 1 . The coil which connect to the metal detector CS209A is embedded in the dedicated fixture. The dedicated fixture, coil and gun tube centerline are collinear. The distance of two detection coils is $d$, and the structure and material are the same.

When the No. $\underline{i}$ projectile have launched and began to close to the first coil, the metal detector external resonant circuit, which composed of the coil and the shunt capacitance, it's $Q$ value decreases and resulting in resonator voltage drop. When the envelope of 
the resonator reaches a certain value, the metal detector's output state turned, until the projectile completely off the coil output state of the metal detector turned again and output an approximate square wave pulse signal. The phase of the signal is $\theta_{i 1}$. When the projectile is completely off the second coil, the second metal detector output the second approximate square wave pulse signal, which phase is $\theta_{i 2}$. The phase difference of the two pulse signals is $\theta_{i e}$.

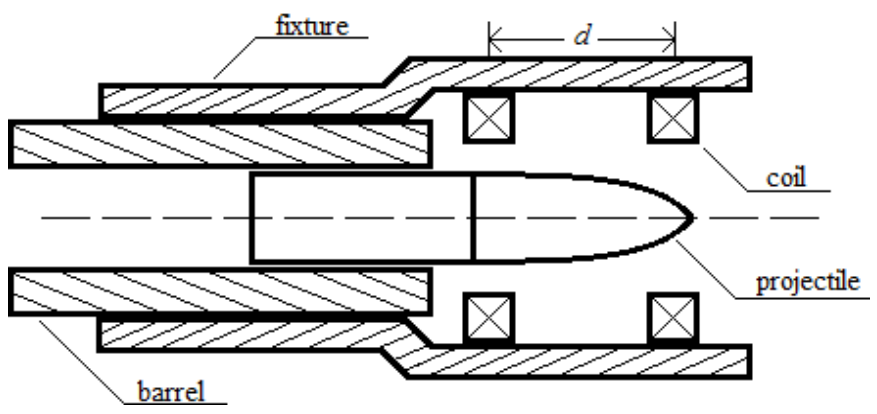

Fig. 1 The structure principle diagram of muzzle velocity measurement system

$\theta_{i e}=\theta_{i 2}-\theta_{i 1}=\frac{d}{V \cdot 2 \pi}$

Two pulse signals are fed into the high speed phase locked loop NE564 and doing phase comparison, and then output a stable voltage value $V_{d}$ which is proportional to the $\theta_{i e}$. There is the decision formula

$V_{i d}=K_{d} \cdot \theta_{i e}$

The $K_{d}$ in the formula is the gain factor of the phase detector (PD), and the unit is $V / \mathrm{rad}$.

Because of the projectile is in the state of flight with high speed, and the distance $\mathrm{D}$ is less than the length of itself, so the projectile's velocity can be approximated thought to be the same when the projectile in the process of passing through the $d$, and the phase difference is fixed in one measurement. Ignoring the Doppler effect. Putting the formula (2) into the formula (1) and get the muzzle velocity $v_{i}$.

$V_{i}=\frac{d \cdot K_{d} \cdot 2 \pi}{V_{i d}}$

\section{TEST PROCEDURE}

First of all, power up the system and firing a projectile. When the projectile flying close to the first coil, the first metal detector CS209A changes in the output state due to external resonator $Q$ value changes, and produced the first pulse signal enter the PPL circuit after shaping. When the projectile close to the second coil, the second metal detector outputs the second pulse signals and enters the PPL circuit after shaping. PPL circuit has characteristics of frequency tracking, which can keep the input and output signal phase difference. The constant phase difference makes the PD in the PPL outputting a voltage $V_{i d}$ which maintain constant. The phase difference of the two pulse signals $\theta_{i d}$ can be calculated by using the voltage value. Using the data acquisition card to collect the $V_{i d}$ and then sent it to the upper computer to calculate the projectile muzzle velocity.

\section{HARDWARE DESIGN}

Design of Metal Close Detector Circuit. The metal close detector circuit built by CS209A is shown in Figure 3 . The main function of the circuit is realized by an oscillator. The oscillator is built by external induction coil-capacitor resonators and the crystal oscillator connect between the OSC and RF pin in order to guarantee the measurement precision. After calculation, this type of rapid rate firing gun fired two type of projectile that produce the phase differential signal frequency does not exceed $30 \mathrm{kHz}$, so selecting the oscillator as the standard $26 \sim 32.768 \mathrm{KHz}$ crystal. Figure 4 shows the relationship between the CS209A demodulation of the voltage and detection distance diagram. In order to ensure the precision, setting the resistance of the crystal oscillator is $7.5 \mathrm{k} \Omega$, and the detection distance is $2.54 \mathrm{~mm}$.

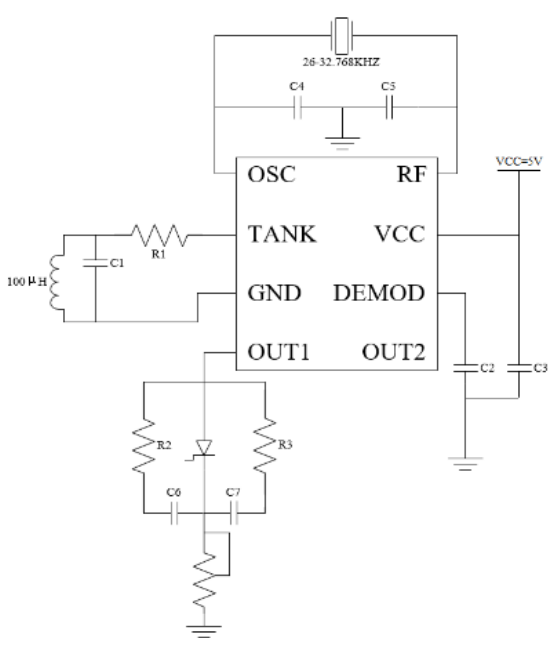

Fig. 3 The circuit of mental close detector

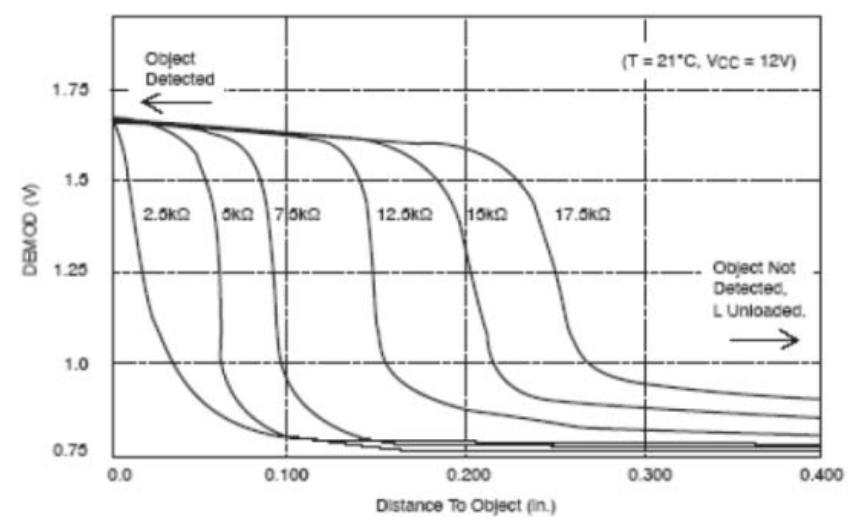

Fig. 4 The relationship between the demodulation of the voltage and detection distance 
Step recovery diode (SRD) with large storage time and minimal transient time. The literature (WANG Zhi-ying, 1989) shows that SRD can shaping rectangular pulse which rise time is less than $800 \mathrm{ps}$, and reduced the pulse rise time amongst the range of $(140+$ 20) ps. In order to get a good transient response performance, using the SRD to shape the output pulse signal at the 4 pin as shown in figure 3.

Phase Detection Circuit Design. Using the analog phase locked loop NE564 of which maximum operating frequency up to $50 \mathrm{MHz}$ to construct the phase detection circuit. The circuit is suitable for testing the muzzle velocity of all the types of projectile. Figure 5 shows the internal circuit of NE564, of which pin $4 \&$ 5 external capacitor to constitute the loop filter that filtering the ripple wave of outputted DC error voltage by the comparator of PD. PD can differentiate two input signal phase difference. The external circuit as shown in Figure 6 . The pin 2 attach to resistance $\mathrm{R} 2$ and rheostat R. Adjusting the value of rheostat $\mathrm{R}$ to change the loop gain. The pin 3 and pin 6 are respectively inputted by two pulse signal from CS209A, which the pulse signal input from pin 3 as a reference signal of pin 6 . When the loop is locked, the phase difference constant and PD maintaining output a constant voltage which being outputted an approximate square wave pulse signal through amplified and DC restored from pin 14.

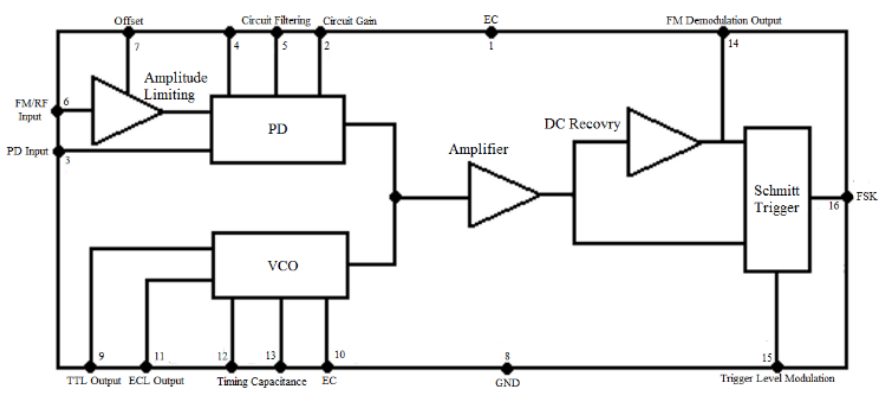

Fig. 5 The internal circuit of NE564

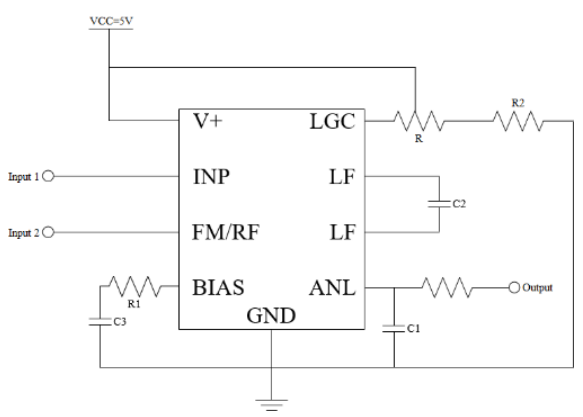

Fig. 6 Design of the circuit of analog PPL

\section{UPPER COMPUTER SOFTWARE DESIGN}

Muzzle velocity calculation software is developed by LabVIEW software. Figure 7 shows the software front panel. After the initialization of the program, first, collect the data received from the serial port, then judge the validity of numerical. Count the projectile number as if the data is valid. Next, calculate the muzzle velocity based on the data acquisition and store the results.

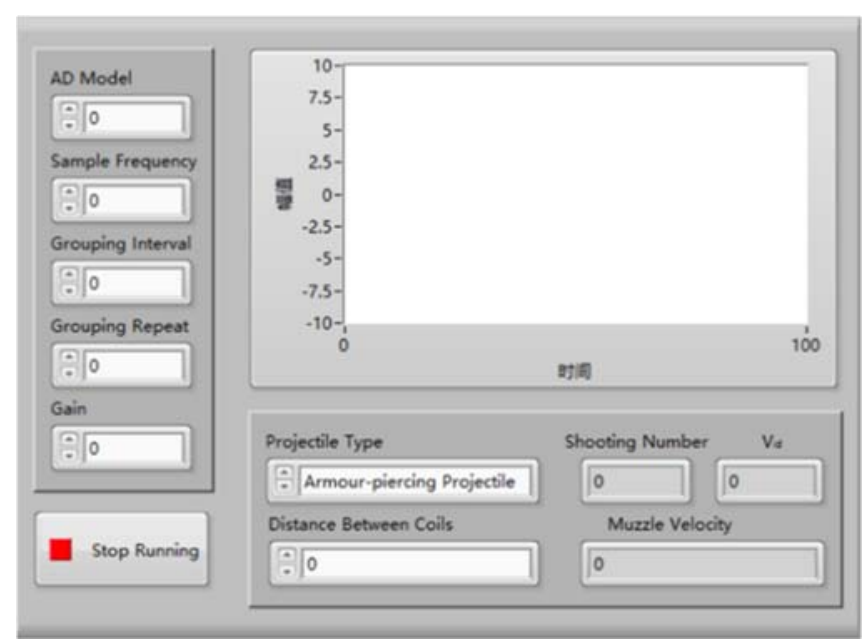

Fig. 7 The front panel of the upper computer software

\section{TESTING RESULTS AND ANALYSIS}

The gun that being tested has a good technical condition and have serviced for a short time. The gun's actual test data of muzzle velocity is $v_{c i}$. To have the qualified inspect for the measurement error of the speed measuring device. Using the average values from test data collected by two velocity radar as the truth $v_{z} 0 i$. The ' $i$ ' is the projectile number.

Under the condition of the confidence level is 1$\alpha=0.9$, setting the sample number of the error check is 18 .

When the gun launch the No.i projectile, the relative measurement error $E_{i}$ for the test muzzle velocity $v_{c i}$ is

$$
\begin{gathered}
E_{i}=\left(V_{c i}-V_{z 0 i}\right) / V_{z 0 i} \\
\text { Velocity error } E \text { is } \\
E=\sqrt{\frac{1}{n} \sum_{i=1}^{n} E_{i}^{2}}
\end{gathered}
$$

Using the hypothesis test method of probability theory and mathematical statistics theory to process the test data of muzzle velocity measurement system and test data from Doppler radar measurement, and then find the measurement error of the acceptance criteria.

One criterion is the measurement error of E should not be greater than the error index $\eta$

$$
E \leq \eta
$$

The above criterion is essentially a normal population variance $N\left(\mu, \sigma^{2}\right)$ test problem. Supposing the samples $E_{1}, E_{2}, \ldots, E_{n}$ are obtained from the normal population $\mathrm{N}\left(\mu, \sigma^{2}\right)$, and the true expect of the normal population $\mu$ is unknown. Using the $\chi^{2}$ test proceed to Hypothesis test

Original hypothesis: $H_{0}: E^{2} \leq \eta^{2}$ 
Alternative hypothesis: $\quad H_{1}: E^{2}>\eta^{2}$

Testing statistic is

$\chi^{2}=\frac{n E^{2}}{\eta^{2}}$

Under a certain confidence level of 1- $\alpha$, by searching the $\chi^{2}$ distribution table to get the measurement error reception area is

$$
\chi^{2}<\chi_{(1-\alpha)}^{2}(n)
$$

Otherwise, the domain is rejected.

Table 1 is a set of measurement error data table. It's easily get the $n=18, E=0.27 \%$ from the table 1 . The measurement error of the measurement system is $\eta=0.25 \%$. Using $\eta$ as the measuring error index of the system, then the test statistic $\chi^{2}$ can be calculated.

$\chi^{2}=\frac{n E^{2}}{\eta^{2}}=20.995$

Table 1 Velocity Measure Error Data

\begin{tabular}{cccccccc}
\hline No & $\mathrm{v}_{0 i} /(\mathrm{m} \cdot \mathrm{s}-1)$ & $\mathrm{v}_{\mathrm{ci}}(\mathrm{m} \cdot \mathrm{s}-1)$ & $\mathrm{Ei}$ & $\mathrm{No}$ & $\mathrm{v}_{0 i} /(\mathrm{m} \cdot \mathrm{s}-1)$ & $\mathrm{v}_{\mathrm{ci}}(\mathrm{m} \cdot \mathrm{s}-1)$ & $\mathrm{Ei}$ \\
\hline 1 & 955.525 & 958.832 & 0.0035 & 10 & 958.762 & 963.747 & 0.0052 \\
2 & 958.832 & 960.921 & 0.0022 & 11 & 961.831 & 963.465 & 0.0017 \\
3 & 964.47 & 965.497 & 0.0011 & 12 & 959.504 & 964.407 & 0.0051 \\
4 & 962.157 & 963.286 & 0.0012 & 13 & 958.423 & 959.073 & 0.0007 \\
5 & 963.747 & 964.179 & 0.0004 & 14 & 964.284 & 962.157 & -0.0022 \\
6 & 963.465 & 959.876 & -0.0037 & 15 & 955.701 & 958.932 & 0.0034 \\
7 & 965.497 & 964.753 & -0.0008 & 16 & 959.762 & 955.525 & -0.0044 \\
8 & 964.179 & 965.585 & 0.0015 & 17 & 958.892 & 958.909 & 0 \\
9 & 959.767 & 961.192 & 0.0015 & 18 & 958.936 & 959.767 & 0.0009 \\
\hline AVG & 960.763 & 961.672 & 0.001 & SD & 3.0312 & 2.859 & 0.0026 \\
\hline
\end{tabular}

Under different conditions of significant level, the testing results are shown in Table 2.

Table 2 The Judgement Result of Velocity Measure Error in Different $\alpha$

\begin{tabular}{l|lrc}
\hline \multirow{2}{*}{ Project } & \multicolumn{4}{l}{ Significant Level } \\
\cline { 2 - 4 } & 0.05 & 0.10 & 0.20 \\
\hline$\chi_{(1-\alpha)}^{-}$ & 24.996 & 22.307 & 19.311 \\
$\chi^{\llcorner}$ & 20.995 & 20.995 & 20.995 \\
Judgement result & qualified & qualified & unqualified \\
\hline
\end{tabular}

The following conclusions can be obtained from the above analysis. The formula (6) can determine the muzzle velocity measurement system and the measurement error index is unqualified. But in the point of view of probability and mathematical statistics, on the level of $\alpha=0.2$ the muzzle velocity measurement system and the measurement error index are qualified. The literature (Wang Ke, 2015) show that the criterion formula (8) is more scientific and reasonable than the formula (6) in the description of the error of the velocity measurement with random. So, it can be considered that the measurement system can achieve the same accuracy of the radar.

\section{CONCLUDING REMARKS}

According to the characteristics of the rapid rate firing gun launching, the paper presents phase method to measure muzzle velocity and basis on that design a muzzle velocity measure system. The overall scheme of the measurement system is designed, then set up the hardware circuit and the program the software. After the test verification and results comparison of the firing test results, it can be seen that the muzzle velocity measurement system is of high feasibility and has the advantages of small volume, simple structure, convenient installation, high precision, real-time collecting data and so on. The muzzle velocity measurement system can adapt to similar rapid firing gun's muzzle velocity measurement, and it's suitable for widespread in the Army. However, the test results show that the measurement system in the state of $\alpha=0.2$ which measurement error is judged to be unqualified, the significant level is slightly smaller. It's proved that the measurement system need to improve the testing accuracy.

\section{REFERENCES}

CHU Ling-yue, GAO Jin, LI Chao-wei. Research on Muzzle Velocity Measurement System Based on Dual Laser Light Screen[J], Journal of Gun Launch\&Control,2010.3:67-70.

HUANG Xue-gong. Study on the Technology of Muzzle Reaction Fuse and Experiment[D]. Nan Jing: Nanjing University of Science and Technology, 2006

LI Xiao-yin, WU Sheng-li XIE Zhi-huai. Research on Projectile Muzzle Velocity Measurement System Based on Laser[J]. Journal of Test and Measurement Technology,

Wang Ke. Research on Velocity Measurement Technology of Coil Target[D]. Xi' an Technological University, 2015.5.25.

WANG Zhi-ying. Nuclear Electronics Principle and Technology[M]. Beijing: the Press of Atomic Energy, 1989.

ZHANG Fu-san, YAO Zhi-jun, ZHANG Pei-zhong. New computation method for gun test and dynamics design[M]. Beijing: National Defense Industry Press, 2013.(in Chinese) 